\title{
Network Architecture for Ultra Low Latency Applications
}

\author{
Patrick Fiati \\ Kwame Nkrumah University of Science and \\ Technology \\ Department of Electrical, Electronic \\ Engineering \\ Kumasi, Ghana
}

\author{
K. Adu Boahen Opare, PhD \\ Kwame Nkrumah University of Science and \\ Technology \\ Department of Electrical, Electronic \\ Engineering \\ Kumasi, Ghana
}

\begin{abstract}
$5 \mathrm{G}$ is the next frontier of innovation for the wireless industry and the broader ICT ecosystem. It is common consensus that $5 \mathrm{G}$ will focus on the breakthroughs to support the expansion and enhancement of mobile internet and Internet of Things (IoT). The application of $5 \mathrm{G}$ in IoT and vertical industries will bring more market space and present business opportunities to operators. In addition, expanded and enhanced mobile internet services will help further improve the consumers experience, strengthen user stickiness and guarantee operators' revenues and profits.
\end{abstract}

\section{Keywords}

IoT, SCMA, OFDMA, MIMO, 5G

\section{INTRODUCTION}

Figure 1 shows.

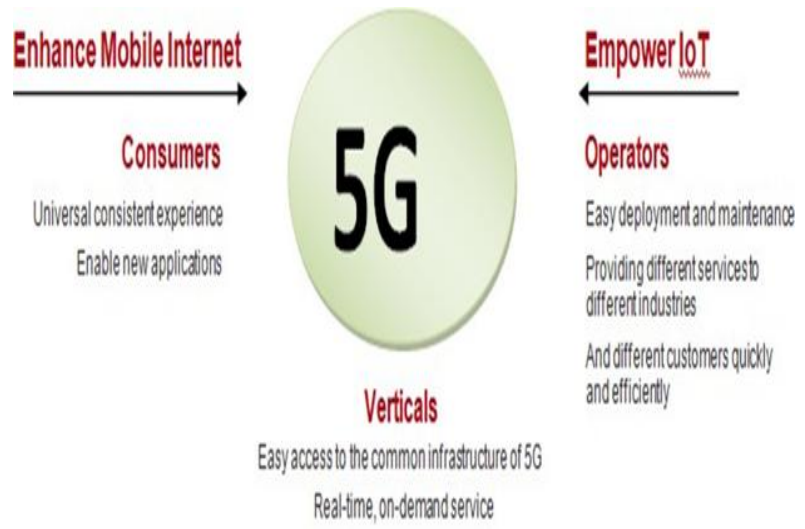

\section{Fioure 1:56 will Carry Many Inoustries and Benefif Sakeholders.}

To adequately support the development of mobile Internet and IoT, 5G networks will increasingly become the primary means of network access for person-to-person and person-tomachine connectivity. This means that $5 \mathrm{G}$ will need to match the diversity of service requirements and service characteristics. Broadband, ultra-low latency, massive connection and ultra-high reliability etc., along with the ability to accommodate various use cases. The strong requirement of a service oriented network to provide better user experience in a flexible, efficient way is raised Examples include extreme broadband, ultra-low latency, massive connection and ultra-high reliability[1] etc., along with the ability to accommodate various use cases. The strong requirement of a service oriented network to provide better user experience in a flexible, efficient way is raised.

\section{5G PERFORMANCE REQUIREMENTS}

Wireless networks will need to match advances in fixed networking in terms of delivered quality of service, reliability and security. It is expected that the $5 \mathrm{G}$ system design will support three orders of magnitude higher capacity per $\mathrm{km} 2$, a hundred times higher data rate, latency of less than $1 \mathrm{~ms}$ across the radio access link, a hundred times more connections (links) and three orders of magnitude lower energy consumption than the current generation of wireless network. Although these performance targets do not need to be met simultaneously, they provide the basis for the Gbit/ s user experience for $5 \mathrm{G}$ networks [2]. The improved performance targets of the overall $5 \mathrm{G}$ system are shown in Figure 2

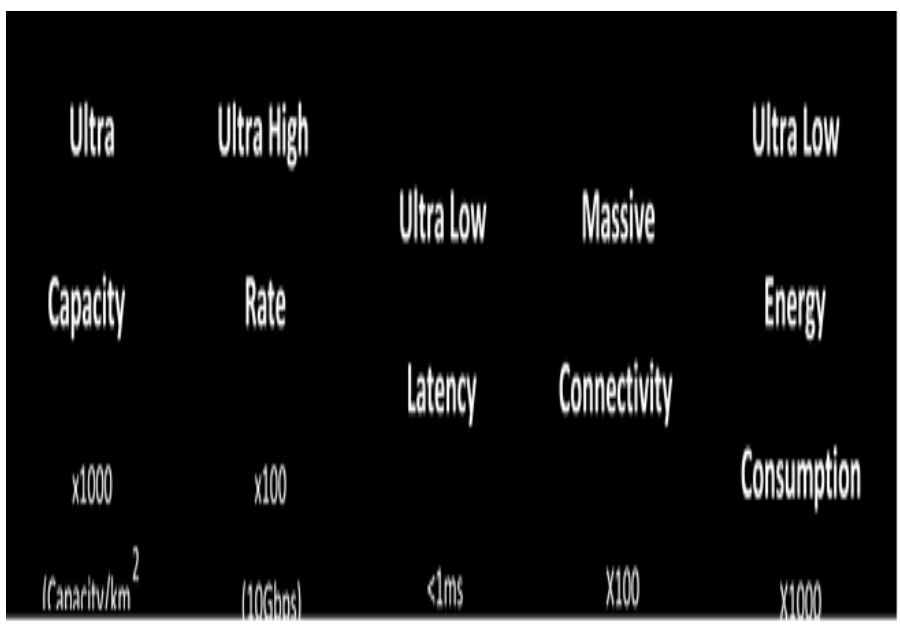

Figure 2: 5G Performance Targets

Based on the requirements, two major challenges should be addressed for the design of the $5 \mathrm{G}$ system:

- The 5G system should be capable of flexible and efficient use of all available spectrums from low band to high band and licensed to unlicensed bands.

- $\quad$ The $5 \mathrm{G}$ system should be adaptable to provide efficient support for the diverse set of service characteristics, massive connectivity and massive capacity. Flexible network design is required to improve spectral efficiency, increase connectivity and reduce latency.

The requirements and challenges will impact on the design of $5 \mathrm{G}$ air interface and the network architecture. In this white paper, the flexible 5G new air interface is explored in details. The viewpoint of network architecture is to be presented in subsequent white papers. 
The 5G air interface framework is built upon two major concepts: software defined flexible air interface and radio access virtualization. In terms of air interface, it should be optimized in the way to support versatile application scenarios. In terms of radio access virtualization, it encompasses self-organization and coordination algorithms that utilize the features, protocols and interfaces to avoid the limitations of the geographic "cell" construct [3].

\section{5G SPECTRUM}

The growing traffic demand necessitates increasing the amount of spectrum that may be utilised by the $5 \mathrm{G}$ systems. High frequency bands in the centimeter wave (cmWave) and millimeter wave (mmWave) range will be adopted due to their potential for supporting wider channel bandwidths and the consequent capability to deliver high data rates.

The new spectrum below $6 \mathrm{GHz}$ is expected to be allocated for mobile communication at the World Radio Conference (WRC) 2015, and the band above $6 \mathrm{GHz}$ expected to be allocated at WRC 2019, as shown in Figure 3.

\section{WRC15}

WRC19

Requirement $>500 \mathrm{MHz}$ for IMT-2020 $45 \mathrm{GHz}$ available

for future Cellular Access and Self-Backhaul

Cellular

$\begin{array}{lllllll}\text { Bands } & 12345610 & 20 & & 30 & 40 \\ & 6070 & 80 & 90 & \text { GH } & \text { Visible Light }\end{array}$

5G Primary bands 5G Complementary Bands for Capacity, $45 \mathrm{GHz}$ available

Figure 3: 5G will aggregate $\mathrm{Sub} 6 \mathrm{GHz}$ and the Bands above $6 \mathrm{GHz}$.

$5 \mathrm{G}$ network is a heterogeneous network which enables the cooperation between lower-frequency wide-area coverage network and high- frequency network. The consensus is higher frequency bands are the complementary bands to $5 \mathrm{G}$ whereas low frequency bands $(<6 \mathrm{GHz})$ are still the primary bands of $5 \mathrm{G}$ spectrum [4].

High frequency also enables unified access and backhaul since the same radio resources is shared. It is expected to use a unified air interface and a hierarchical scheduling for both radio access and backhaul which enables flexible backhauling and low-cost ultra dense networking (UDN).

Future radio access may also employ bands with different levels of access regulation including exclusive licensed, nonexclusive licensed and unlicensed bands. The 5G system treats both the licensed and unlicensed spectrum in a flexible, unified air interface framework.

\section{5G FLEXIBLE NEW AIR INTERFACE}

Unified new air interface with flexibility and adaptability is proposed to meet these requirements. New air interface consists of building blocks and configuration mechanisms such as adaptive waveform, adaptive protocols, adaptive frame structure, adaptive coding and modulation family and adaptive multiple access schemes. With these blocks and mechanisms, the air interface is able to accommodate the future wide variety of user services, spectrum bands and traffic levels.

Key technology components, as shown in Figure 4-1, include a new waveform technology Filtered-OFDM (FilteredOrthogonal Frequency Division Multiplexing), a new multiple access technology SCMA (Sparse Code Multiple Access), a new channel code Polar Code, the full-duplex mode and massive MIMO technology. The new air interface design can effectively improve spectral efficiency, increase connectivity, and reduce latency, thus facilitating the deployment of customized scenarios applied to the IoT and for high bandwidth-consuming scenarios such as virtual reality.

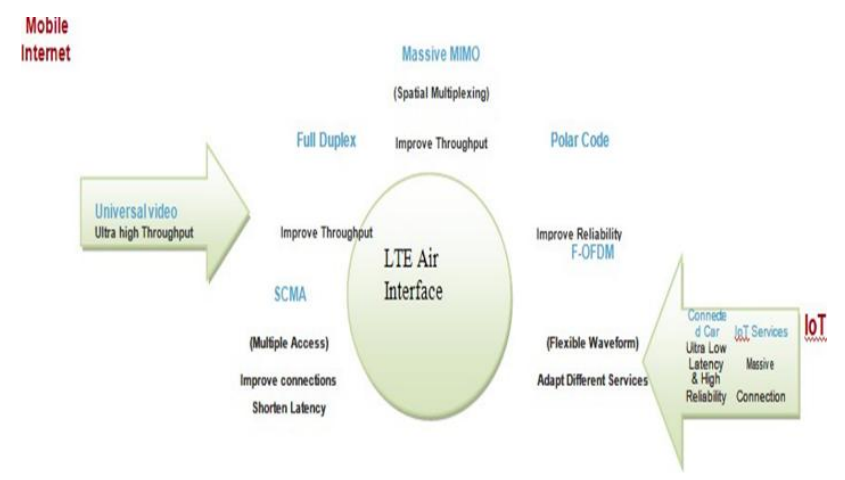

One arinterface fis many applications withigh flexibility, at least $3 x$ spectum efficiency improvement

Fioure 4-1: New air interface components.

The new air interface exploits two-level non-orthogonality to maximize the spectrum efficiency, the number of connected devices and to provide flexibility to support vastly diverse services. Filtered OFDM allows inter-subband nonorthogonality while SCMA enables intra-subband nonorthogonality.

Filtered - OFDM

Filtered-OFDM is one element of fundamental waveform technology to support different waveforms, multiple access schemes and frame structures based on the application scenarios and service requirements simultaneously. It can facilitate the co-existence of different waveforms with different OFDM parameters as shown in Figure 4-2. In this figure different sub-band filters are used to create OFDM subcarrier groupings with different inter-sub-carrier spacing, OFDM symbol durations and guard times. By enabling multiple parameter configurations, filtered-OFDM is able to provide a more optimum parameter choice for each service group and hence better overall system efficiency.

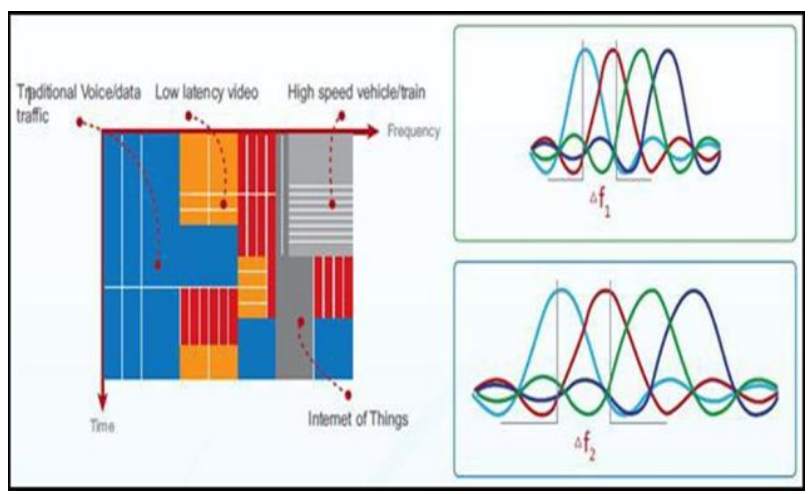

Figure 4-2: Filtered-OFDM enables flexible waveform parameters 


\section{Sparse Code Multiple Access}

Sparse code multiple access (SCMA) is another waveform configuration of the flexible new air interface. This nonorthogonal waveform facilitates a new multiple access scheme in which sparse codewords of multiple layers of devices are overlaid in code and power domains and carried over shared time-frequency resources. Typically, the multiplexing of multiple devices may become overloaded if the number of overlaid layers is more than the length of the multiplexed codewords.

However, with SCMA, overloading is tolerable with moderate complexity of detection thanks to the reduced size of the SCMA multi-dimensional constellation and the sparseness of SCMA codewords. In SCMA, coded bits are directly mapped to multi-dimensional sparse codewords selected from layerspecific SCMA codebooks.

The complexity of detection is controlled through two major factors. One is the sparseness level of codewords, and the second is the use of multi-dimensional constellations with a low number of projection points per dimension. An example of device multiplexing with a low projectioncodebook and the resulting constellation mapping is shown in Figure 4-3. A device's encoded bits are first mapped to a codeword from a codebook. In the example, a codeword of length 4 is used. The low projection codebook has a reduced constellation (from 4 points to 3 points). Furthermore, each point (e.g. "00") has non-zero component only in one tone. A codebook with one non-zero component is a zero- PAPR codebook.

Furthermore, a blind multi-device reception technique can be applied to detect device activities and the information carried by them simultaneously. With such blind detection capability, grant-free multiple access can be supported. Grant-free multiple access is a mechanism that eliminates the dynamic request and grant signaling overhead. It is an attractive solution for small packets transmission. SCMA is an enabler for grant-free multiple access. Due to these benefits, SCMA can support massive connectivity, reduce transmission latency and provide energy saving.

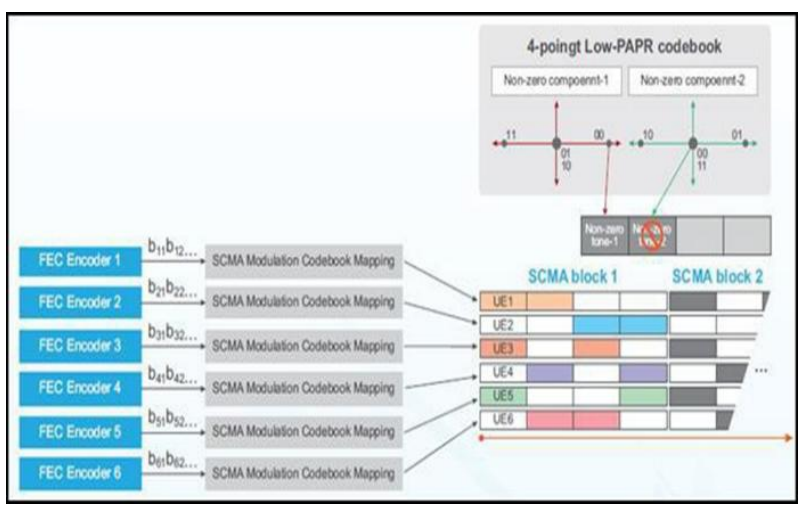

Figure 4-3: SCMA multiplexing and low projection codebook constellation

\section{Polar Codes}

Polar codes are a major breakthrough in coding theory. They can achieve Shannon capacity with a simple encoder and a simple successive cancellation (SC) decoder when the code block size is large enough. Polar codes have brought significant interests and a lot of research work has been done mainly on code design and decoding algorithm. One of the most important decoding algorithms is the SC-list decoding which can perform as well as the optimal maximumlikelihood (ML) decoding with a list size of 32 for moderate code block sizes. A lot of performance simulations show that Polar codes concatenated with cyclic redundancy codes (CRC) and an adaptive SC-list decoder can outperform turbo/LDPC (Low Density Parity Check) codes for short and moderate code block sizes. Polar code has better performance than all the codes currently used in the 4G LTE systems, especially for short code length, thus it is considered as a perfect candidate for the FEC (Forward error correction) module in 5G air interface design [5].

\section{Massive-MIMO}

Massive MIMO makes a clean break with current practice through the use of large-scale antenna systems over network and devices. As one of the most promising ingredients of the emerging 5G technology, massive MIMO is a commercially attractive solution since 100x higher efficiency is possible without installing 100x more base stations.

The progress on omni-directional beam with low power, low PAPR and flexible beam adjustment for mobility UE tracking is enabling the theoretical concept to commercial deployment reality in diverse scenarios, such as macro, micro, suburb and high-rise.

\section{Full Duplex}

Full-Duplex breaks the barrier of today's communications by supporting bi-directional communications without time or frequency duplex.

By transmitting and receiving at the same time and on the same frequency, Full-Duplex has the potential to double the system capacity and reduce the system delay.

\section{RADIO ACCESS VIRTUALIZATION}

5G network densification. The virtual cell concept removes the traditional cell boundary for the device and Provides a consequent reduction of the detrimental "cell-edge experience" by the device.

\section{Elimination of cell boundaries}

Traditionally devices associate with a cell as a consequence the link performance may degrade as a device moves away from the cell center. In a virtualized device centric network, the network determines which access point(s) are to be associated with the device. The cell moves with and always surrounds the device in order to provide a cell-center experience throughout the entire network. The elimination of the device's view of the cell boundary is illustrated in Figure 5 .

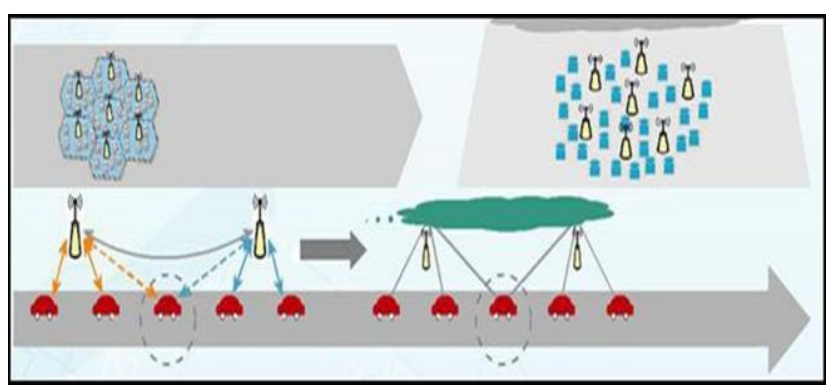

Device-centric Access Point Optimization

Each device is served by its preferred set of access points. The 
actual serving set for a device may contain one or multiple access points and the device's data is partially or fully available at some or a small set of potential serving access points. The access point controller will accommodate each device with its preferred set and transmission mode at every communication instance while considering load and Channel State Information (CSI) knowledge associated with the access points.

\section{Network-assisted device Cooperation}

An important factor in determining and updating potential and actual serving access point sets is the possibility of cooperation among neighboring devices and the nature of such cooperation. The density of neighboring devices and the capability for device to device (D2D) connectivity provides the opportunity for device cooperation in transmission/reception.

The access point controller can schedule the devices benefiting from the device cooperation and manage factors such as cooperation collision, security and privacy restrictions, and cooperation incentive. A network assisted device cooperation results in better virtualization by providing more possible transmission paths from network to the target devices.

\section{CONCLUSIONS}

The future network will focus on the different business applications and user experience other than just the pursuit of the greater bandwidth and volume. This will raise the requirement to build service oriented networks to quickly and efficiently respond to user needs, as well as to offer consistent and high-quality services for different use cases.
This paper has outlined an overview of $5 \mathrm{G}$ air interface design including the key concepts of air interface adaptation and radio access virtualization. Radio access virtualization technologies can provide the best transmit and receive conditions to users while flexible new air interface selects the best sets of air interface technologies on the wireless links. These two components together can bring the best user experience in the $5 \mathrm{G}$ wireless network. The goal is to design an air interface that is adaptable to the diverse services, applications and devices of the future, scalable to support massive connectivity and massive capacity and intelligent to adapt to the entire locally available spectrum.

\section{REFERENCES}

[1] 5G Network a New Look into the Future: Beyond all Generation Networks Sidhartha Sankar Sahoo, Malaya Kumar Hota, Kalyan Kumar Barik.

[2] A Research based study on Evolution of cellular generations (5G), Amity University (july 2014)

[3] A Research based study on Evolution of Cellular Generations (5G)" Payal1, Bhawna Dhruv2, Praveen Kumar3 M.Tech, (CS\&E).

[4] David Ott, ShilpaTalwar, Intel Labs, University Research Office, "Exploring Next Generation Wireless (5G): Transforming the user Experience", 2013.

[5] Dr. Anwar M Mousa (2012), "Prospective of Fifth Generation Mobile Communications", International Journal of Next-Generation Networks (IJNGN), Vol. 4, No. 3, September. 